\title{
Poster Workshop
}

\section{Breast Cancer}

75 poster worshop

Brachytherapy boost may improve local control in very young patients treated with breast conservation

G. van Tienhoven ${ }^{1}$, G.M. MCColl, ${ }^{1,2}$, E.D. Geijsen ${ }^{1}$, C.C.E. Koning

${ }^{1}$ Academic Medical Center, Radiation Oncology, Amsterdam, The Netherlands

${ }^{2}$ Universitair Medisch Centrum St Radboud, Radiation Oncology, Nijmegen, The Netherlands

Introduction: Very young age is a risk factor for local control in breast conservation. To investigate if type of boost treatment may influence this, all patients of 40 years or younger treated in the Academic Medical Centre (AMC) with long term follow-up were analyzed.

Patients \& Methods: Of 83 patients treated between 1986 and 1998,5 were lost to follow up $(6 \%)$. Of the remaining 78 patients median follow up was 78 months, 54 received a Brachy Therapy (BT) boost and 24 an external Beam (EB) boost. BT boost was applied using a two plane rigid needle implant during operation with open excision wound. Low Dose Rate or Pulsed Dose Rate afterloading techniques were used. EB was mainly performed using 8-14 MeV Electron beams. The choice for EB boost was made mainly for logistic reasons (referral after surgery).

Results: Seventeen $(21.8 \%)$ patients experienced a local recurrence. A local recurrence was observed in $15 \%(n=8)$ of the BT group and $37.5 \%(n=9)$ of the EB group. The crude fiveyear local recurrence rate was $11 \%(B T)$ and $16.7 \%(E B)$. The actuarial local control differed significantly $(p=0.03)$. The actuarial 5 year survival rate was $82 \%$ in both groups. Cosmetic results were comparable in both groups.

Conclusion: Although these results should be interpreted with caution because of the small numbers and possible biases due to the retrospective nature of the study, they suggest that long term local control may be improved using (intra operative) brachytherapy in these very young patients.

\section{6 poster worshop}

A randomized study of immediate vs delayed radiotherapy in patients receiving CMF chemotherapy after conservative surgery for breast cancer

\section{G. Arcangeli, P. Pinnarò, C. Giordano, M. Messina}

Regina Elena Cancer Institute, Radiation Oncology, Roma, Italy Introduction: there are still controversies on optimal sequence of chemotherapy and radiotherapy in patients who undergo a conservative surgery for mammary carcinomas. The purpose of this study was to compare two different timing of radiation treatment in patients who underwent a conservative surgery and who were planned to receive adjuvant CMF chemotherapy.

Material and methods: a total of 206 patients, operated in our Institution of quadrantectomy plus axillary clearance for an invasive carcinoma of the breast, in whom six cycles of adjuvant CMF (cyclophosphamide $600 \mathrm{mg}$ per square meter i.v. on days 1 and 8 of each cycle, methotrexate $40 \mathrm{mg}$ per square meter i.v. on days 1 and 8 of each cycle an $5 \mathrm{Fu} 600 \mathrm{mg}$ per square meter i.v. on days 1 and 8 , repeated 28 days intervals) were previously and independently planned by medical oncology, were randomized to receive an immediate (i.e. treatment initiated within 2 months from the day of quadrantectomy) or a delayed radiotherapy (i.e. treatment started within 6 to 7 months from the day of surgery). Patients were stratified for age $(\leq 45 \mathrm{yr}$ vs $>45 \mathrm{yr}$ ), $\mathrm{pT}$ (T1 vs T2) and $\mathrm{pN}$ (N0 vs N1). All patients began CMF within 2 months from surgery. Radiotherapy was delivered only to the whole breast by means of tangential fields to a dose of 50Gy in 20 fractions in 4 weeks, followed by an electron boost of 10 to 15 Gy in 4 to 6 fractions to the tumor bed. Inclusion criteria were invasive carcinomas of the breast, negative surgical margins, no distant metastases, no previous radiotherapy or chemotherapy, age $\leq 76$ years, no previous malignant tumors, written consensus.

Results: all patients completed the planned radiotherapy. No evidence of an increased risk of toxicity was observed in the immediate radiotherapy with respect to the delayed arm. The compliance of the treatment was excellent in both arm groups. No difference in radiotherapy duration between the two groups was observed. The mean dose-intensity of all drugs was not different between the two arms. After a median follow-up of 54 months, no difference in five-years event-free survival was observed in the two groups.

77 poster workshop

DCIS II trial: a randomised trial testing observation (no radiotherapy) against radiotherapy in women with low-risk completely excised oestrogen receptor (ER) positive ductal carcinoma in situ (DCIS) of the breast on adjuvant endocrine therapy

J. Yarnold ${ }^{1}$, N. Bundred ${ }^{2}$, J. Dewar ${ }^{3}$, W. George ${ }^{4}$, J. Haviland $^{5}$, C. Dawson ${ }^{5}, J$. Bliss ${ }^{5}$

${ }^{1}$ Royal Marsden NHS Trust, Department of Academic Radiotherapy, Sutton, United Kingdom

${ }^{2}$ South Manchester University Hospital, Department of Surgery, Manchester, United Kingdom

${ }^{3}$ Ninewells Hospital, Department of Radiotherapy and Oncology, Dundee, United Kingdom

${ }^{4}$ Western Infirmary, Department of Surgery, Glasgow, United Kingdom

${ }^{5}$ Institute of Cancer Research, Clinical Trials and Statistics Unit, Sutton, United Kingdom

Introduction: DCIS has become an increasingly common diagnosis following the advent of mammographic screening. Generally, local treatment comprises either simple mastectomy alone or complete microscopic tumour excision and radiotherapy. Endocrine treatment is usually given to those with ER +ve disease. The purpose of the trial is to identify the group of patients who can be spared radiotherapy. This trial aims to recruit 2000 women who have had complete surgical excision of low risk ER positive DCIS. All patients will receive adjuvant endocrine treatment and will be randomised to observation or radiotherapy.

Methods: Eligible patients will be identified in multi-disciplinary meetings. Clinicians will discuss the trial with the patient and a dedicated nurse will support the patient and supply additional information as necessary. If willing, patients can also take part in the biomarker study and /or the quality of life study. In centres also taking part in the IBIS II (DCIS) trial (blinded study of anastrozole versus tamoxifen), patients can be randomised to both trials. Patients not eligible for, or not willing to take part in, IBIS II (DCIS) trial will receive tamoxifen.

Aims: The primary aim of the trial is to test the effects of avoiding radiotherapy in terms of ipsilateral tumour relapse (invasive and DCIS) and quality of life, following complete microscopic tumour excision in the presence of adjuvant 
systemic endocrine therapy in patients with low-risk ER positive DCIS of the breast. Other aims are to identify the minimum surgical margins required to prevent local recurrence, and to identify molecular markers that predict for ipsilateral tumour recurrence with or without radiotherapy.

Conclusions: It is intended that over 100 centres in the UK, together with centres in Ireland, Australia and New Zealand will participate in the trial. We anticipate the first centres will start recruitment in July 2004. The British Association of Surgical Oncology (BASO) and the NCRI Breast and Radiotherapy Clinical Studies Groups support the trial and funding has been secured from Cancer Research UK

\section{8 poster workshop}

Radiotherapy administration and neutropenic events in five European audits of adjuvant breast cancer chemotherapy C. Jackisch ${ }^{1}, M$. Schwenkglenks ${ }^{2}, M$. Constenla ${ }^{3}$, R. Leonard ${ }^{4}$, R. Paridaens ${ }^{5}$, T.D. Szucs, A. Bosly ${ }^{6}$, R. Pettengell, for the Impact of Neutropenia in Chemotherapy - European Study Group (INC-EU)

${ }^{1}$ University Hospital Marburg, Department of Gynaecology, Marburg, Germany

${ }^{2}$ European Center of Pharmaceutical Medicine, Basel, Switzerland

${ }^{3}$ Complexo Hospitalario de Pontevedra, Pontevedra, Spain

${ }^{4}$ South West Wales Cancer Institute, Swansea, UK

${ }^{5}$ University Hospital Gasthuisberg, Leuven, Belgium

${ }^{6}$ Mont-Godinne, Belgium,

'London, UK

Background: There are few European data on the risk factors and consequences of chemotherapy (CT)-induced neutropenic events (NE) in the adjuvant breast cancer setting. The impact of breast radiation is rarely addressed in this context.

Materials and methods: Data from five retrospective European audits were combined into a single dataset of individual observations. NE were defined as neutropenia-related hospitalisation; CT dose reduction $\geq 15 \%$; or CT dose delay $\geq 7$ days. Analysis focused on NE risk factors and on the occurrence of low average relative CT dose intensity (ARDI < $85 \%$ ). Multivariate adjusted odds ratios (ORs) were calculated by robust multiple logistic regression.

Results: Information on whether CT administration was accompanied by concomitant radiotherapy (RT) was available for 2'409 patients diagnosed between 1983 and 2001. They had a mean age at diagnosis \pm SD of $51.5 \pm 11.3$ years. They were post-menopausal in $52 \%$ and hormone receptor positive in $64 \%$. The spread of disease was $18 \%$ stage I, $65 \%$ stage II, $17 \%$ stage III. CT regimens were CMF-based in $58 \%$, anthracycline-based in $40 \%$, taxane-based in $1 \%$ and other regimens in $2 \%$. Colony-stimulating factors were used in $12 \%$. Any RT was reported in $52 \%$, prior RT in $5 \%$, concomitant RT in $32 \%$, and subsequent RT in $16 \%$. There was concomitant RT in $40 \%$ of the patients with CMF-based regimens, $22 \%$ of those with anthracycline-based, $29 \%$ of those with taxane-based and $18 \%$ of those with other regimens $(p<0.0005)$. Seventy patients receiving $R T$ within a sandwich schedule, mostly in combination with CMF-based CT, were included here.

NE were seen in $20 \%$ of patients. They occurred in $17 \%$ of those without vs $27 \%$ of those with concomitant RT $(p<$ 0.0005 ). Logistic regression showed NE to be independently associated with concomitant RT; age; number of CT cycles planned; cycle duration; and regimen type (Table 1). ARDI $\leq$ $85 \%$ was seen in $16 \%$ of patients. It occurred in $14 \%$ of those without vs $21 \%$ of those with concomitant RT ( $p<0.0005$ ). Exclusion of the patients with a pre-planned interruption of $\mathrm{C} T$ did not alter these results.
Conclusions: Concomitant RT administration was an independent risk factor for NE occurrence and impacted negatively on $\mathrm{CT}$ dose intensity in patients receiving adjuvant breast cancer $\mathrm{CT}$. Increased protective measures may be required when this risk factor is present.

\begin{tabular}{|c|c|c|}
\hline Influence & OR $(95 \% \mathrm{Cl})$ & p-value \\
\hline Concomitant RT & $1.46(1.17-1.82)$ & 0.001 \\
\hline Age & $1.01(1.00-1.02)^{* *}$ & 0.021 \\
\hline No. of CT cycles planned & $1.29(1.19-1.39)^{\star * *}$ & $<0.0005$ \\
\hline Cycle length & $0.96(0.92-1.00)^{* * * *}$ & 0.045 \\
\hline Anthracycline-based CT & $0.60(0.45-0.81)^{\star \star \star \star *}$ & 0.001 \\
\hline Taxane-based CT & $1.74(0.09-1.21)^{\star * * * *}$ & 0.346 \\
\hline Other CT & $0.34(0.24-1.63)^{\star \star \star \star \star}$ & 0.095 \\
\hline \multicolumn{3}{|c|}{$\begin{array}{l}\mathrm{N}<2 \times 209 \text { due to missing values spread over variou } \\
\text { independent variables }\end{array}$} \\
\hline \multicolumn{3}{|c|}{ Per additional year of age } \\
\hline \multicolumn{3}{|c|}{ Per additional cycle planned } \\
\hline \multicolumn{3}{|c|}{ Per additional cycle day } \\
\hline \multicolumn{3}{|c|}{ Compared to CMF-based CT } \\
\hline
\end{tabular}

79 poster workshop

IORT in breast cancer as boost: preliminary results of a pilot randomized study on use of IORT for stage $I^{\circ}$ and $I^{\circ}$ breast cancer

A. Ciabattoni ${ }^{1}$, G. Fortuna ${ }^{1}$, V. Ciccone ${ }^{1}$, S. Drago ${ }^{2}$, G. Grassi ${ }^{2}$ R. Consorti, A. Petrucci

${ }^{1}$ U.O. Radiotherapy, Ospedale S. Filippo Neri, Rome, Italy

${ }^{2}$ U.O. General Surgery, Ospedale S.Filippo Neri, Rome, Italy

${ }^{3}$ U.O. Medical Physics, Ospedale S.Filippo Neri

Introduction: The use of a dedicated mobile linear accelerator makes the IORT procedure quicker and easier. To compare the efficacy on local control of IORT boost after quadrantectomy versus conventional treatment with external electron beam boost a randomized study was carried on. Preliminary results in terms of local control, esthetic evaluation and toxicity were analyzed.

Methods Since April 1999 a pilot randomized study in women with T1-T2 breast cancer was carried on in S.Filippo Neri Hospital in Rome, Italy. All patients underwent conservative surgery and were randomly assigned to receive IORT (10 Gy) and post-operatively external radiotherapy (50 Gy) or postoperatively external radiotherapy $(50 \mathrm{~Gy})$ plus a $10 \mathrm{~Gy}$ external beam boost. At the end of March 2004234 patients have been randomized, 122 in IORT arm (with 127 treatments for 5 bilateral neoplasms) and 112 in non-IORT arm. A mobile linear accelerator Novac7 (Hitesys SPA) was used and a dose of 10 Gy was delivered to PTV1 including tumor volume with a radial margin of 2 centimeters. Electron boost was performed with 6 $12 \mathrm{MeV}$ energy beams, $10 \mathrm{Gys}$ dose with one direct field on the scar area and the use of blocks. Clinical valuation of the patients was regularly made. Well balanced patient's characteristics were observed in the two arms about staging, size of tumor, histology, grading, systemic chemotherapy, surgical margins.

Results: In IORT arm six cases of wound infection (4,7\%) as acute toxicity (not significantly higher than non IORT controls) and 9 seromas $(7,1 \%)$, regressed in $3-6$ months, as late toxicity 